\title{
BMJ Open Music-based interventions to address well-being in people with a vision impairment: protocol for a scoping review
}

\author{
Nurbanu Somani, ${ }^{1}$ Eldre Beukes, ${ }^{1}$ Alexander Street,${ }^{2}$ Rosie Lindsay, ${ }^{1}$ \\ Peter M Allen (1D ${ }^{1}$
}

To cite: Somani N, Beukes E, Street A, et al. Music-based interventions to address wellbeing in people with a vision impairment: protocol for a scoping review. BMJ Open 2022;12:e054268. doi:10.1136/ bmjopen-2021-054268

- Prepublication history for this paper is available online To view these files, please visit the journal online (http://dx.doi org/10.1136/bmjopen-2021054268).

Received 10 June 2021 Accepted 11 February 2022

Check for updates

(c) Author(s) (or their employer(s)) 2022. Re-use permitted under CC BY-NC. No commercial re-use. See rights and permissions. Published by BMJ.

${ }^{1}$ Vision and Hearing Sciences Research Centre, Anglia Ruskin University, Cambridge, UK ${ }^{2}$ Cambridge Institute for Music Therapy Research, Anglia Ruskin University, Cambridge, UK

Correspondence to Professor Peter M Allen; peter.allen@anglia.ac.uk

\section{ABSTRACT}

Introduction Music-based interventions are used to improve well-being in individuals who are psychologically vulnerable and have long-term illnesses. To date, no study has systematically assessed the literature on music-based interventions aimed at improving well-being in people who have a vision impairment (VI). The purpose of the current protocol is to provide the methodology for a scoping review, to explore the therapeutic outcomes and strategies used in music-based interventions aimed specifically at people with a Vl.

Methods and analysis This scoping review protocol was developed according to the Joanna Briggs Institute methodology and reporting will follow the Preferred Reporting Items for Systematic Reviews and MetaAnalyses Extension for Scoping Reviews checklist and guidelines. The anticipated start date for this study was July 2021. The proposed review will include studies that use music therapeutically as part of a treatment intervention for all VI populations. Studies that meet the inclusion criteria with regards to population, concept and context will be included. Electronic database searches will be conducted independently by two researchers and include MEDLINE, EMBASE, CINAHL Plus, PsycINFO and Web of Science. Further searches will include the reference lists of included studies and grey literature. A narrative synthesis will be conducted to map out the types of therapeutic music interventions undertaken and to compare therapeutic outcomes.

Ethics and dissemination As the methodology of this study consists of collecting data from publicly available articles, it does not require ethics approval. The findings of the planned scoping review are important to guide the development of future interventions, or strategies, that will attempt to use music to improve well-being in people with a VI. The results will be disseminated through a peerreviewed publication and conference presentations.

\section{INTRODUCTION}

World Health Organisation (WHO) estimates there are over 1 billion people globally who have an irreversible vision impairment (VI). ${ }^{1}$ Growth in population and increase in lifespan indicate that the number of people with VI is set to rise. ${ }^{2} \mathrm{~A}$ VI is often associated
Strengths and limitations of this study

- The study will use best practice methods to conduct a scoping review.

- Publications written in all languages will be considered for inclusion.

- Any type of clinical trials, comparative, evaluative and observational studies, will be eligible.

- A potential limitation could be the small number of eligible articles in the literature.

- A limitation of this review is that it will not include a quality assessment as this does not form part of the scoping review remit.

with reduced psychological and emotional well-being, ${ }^{3-10}$ due to the limitations imposed by the loss of sight, such as greater difficulty reading, writing, comprehending non-verbal cues and following conversations. ${ }^{11-13}$ The restrictions faced by people with a VI, particularly if sight loss is acquired, may lead to elevated psychological strain due to the significant adaptations needed for the individual to adjust to their new lifestyle and identity. ${ }^{14}$ This adaptation process may cause depression, ${ }^{15-18}$ emotional distress, ${ }^{17}$ anxiety, ${ }^{19-22}$ feelings of loneliness, ${ }^{23}$ social isolation ${ }^{23}$ and a loss of sense of belonging. ${ }^{2425}$ This may be a result of increased social interaction barriers, ${ }^{26}{ }^{27}$ for example, no longer being able to recognise faces ${ }^{2627}$ which can feel embarrassing, undermine confidence when in social groups ${ }^{25}$ and as a result lead to withdrawing from social situations. $^{24}$

Presently, psychosocial well-being interventions to help with adjustment to living with VI are limited. ${ }^{28-31}$ Additionally, the psychosocial needs of people with a VI have been exacerbated amidst the COVID-19 pandemic, rendering those with a moderate to severe VI three times more likely to experience increased loneliness than those with mild to 
no VI. ${ }^{32}$ One prospective way to address the psychosocial well-being of people with a VI can be through music interventions. Music can activate widespread neural networks, including emotional, cognitive and motor. ${ }^{33-37}$ Engaging in music interventions can instil a sense of belonging and feelings of interpersonal attachment which can offset loneliness. ${ }^{38-41}$ Music listening can also be used to alter arousal to help manage psychological conditions by reducing levels of anxiety, stress and depression. ${ }^{42}{ }^{43}$ In recent years, researchers have developed algorithms to map music in relation to arousal, enabling automated listening systems for arousal adjustment, linked to the listener's heart rate and other biomarkers.$^{43} 44$ Therefore, it is possible that music interventions can be used in VI populations to promote social cohesion, interpersonal communication ${ }^{12} 13$ and for relaxation. Listening to calming music has been used during medical treatment for open-angle glaucoma ${ }^{45-47}$ and during cataract surgery. ${ }^{48-50}$ The most recent review of music interventions for people with a VI was conducted by Park et al. ${ }^{51}$ Although this study added some value in terms of music intervention trends for educational purposes, it excluded studies that used music for relaxation and did not focus on the therapeutic outcomes of those studies to promote psychosocial well-being. Moreover, there have been additional music intervention studies conducted with people who have a VI since the aforementioned review was published. Therefore, it would be of merit to conduct an in-depth investigation into how music interventions could be best utilised for people with a VI to promote psychosocial well-being, and explore how the effects of music may vary.

A scoping review is the most useful tool to examine and investigate a new research topic that has not been fully established in the literature. ${ }^{52}$ Thus, the aim of this scoping review is to synthesise the key concepts and outcomes of studies which have used music as a therapeutic treatment for people with a VI. This will include comparing the contexts in which the music therapies have been used, as this may provide insights into the equitability of music interventions for health and well-being based on the ethnicity of recipients and those delivering the interventions.

An additional aim will be to investigate if there were any accessibility challenges that may have hindered the participation of people with VI in the intervention. This is important to highlight, as often people with VI are not always able to access face-to-face interventions because of constraints related to transport, geographical location of clinic and/or finances. ${ }^{53}$ Similarly, we will also review if any special arrangements and/or accessibility protocols were utilised at the intervention setting and during the treatment to address the challenges people with VI may face when navigating unfamiliar settings (both online and in-person) and conversing with people who may not have the skills to adapt their communication style appropriately for people with VI. ${ }^{54}$

The key areas to be identified are music-based activities, music listening and associated technology, and music therapy protocols designed for people who have a VI. The scope, extent, treatment tolerance, adherence, fidelity and range of research activity undertaken in this area will be examined using the Checklist for Reporting Music-based Interventions. ${ }^{55}$ This has been used effectively in previous studies as a tool to report the quality of the music intervention research in terms of clinical relevance and rigour. ${ }^{56-58}$ Additionally, the accessibility and useability of the music-based intervention will be reviewed. This information will provide insights which will inform the design of future music interventions. It will also highlight the psychosocial needs, and contribute towards the equitability of access to such interventions across VI populations.

\section{Scoping review questions}

The research questions that will guide the scoping review are presented in table 1.

\section{METHODS}

This protocol will follow the methodology manual, published by the Joanna Briggs Institute (JBI) methodology for scoping reviews ${ }^{59}$ and the Preferred Reporting Items for Systematic Reviews and Meta-Analyses for Scoping Reviews (PRISMA-ScR) extension for scoping reviews. ${ }^{60}$ A limitation of this review is that it will not include a quality assessment as this does not form part of the scoping review remit. ${ }^{61}$ This scoping study is anticipated to take place between July 2021 and January 2022. Any changes made to this published protocol will

\section{Table 1 Scoping review questions}

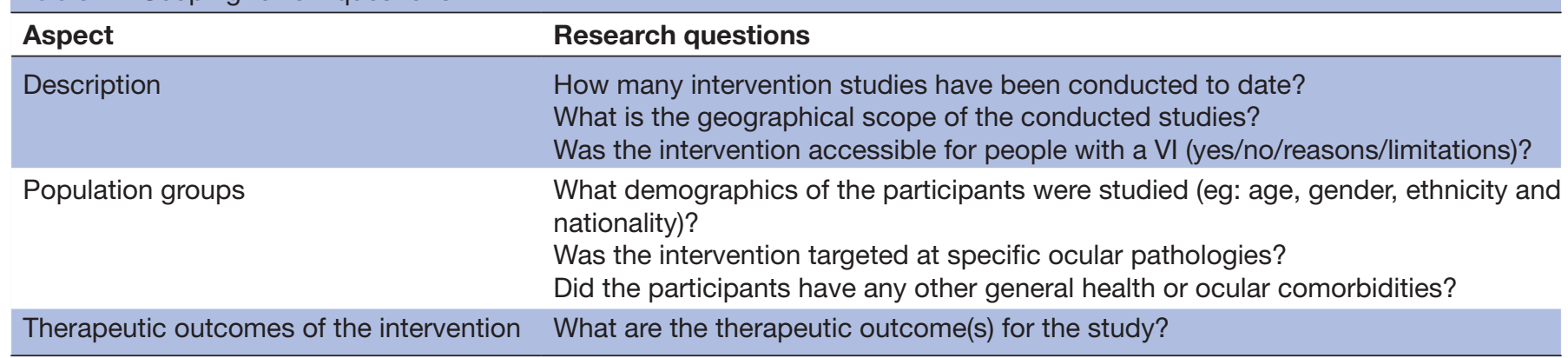


be recorded and referred to in the full publication of the scoping review. A search on Cochrane Reviews, JBI Evidence Synthesis and Prospero showed no current or ongoing scoping review on this topic. The format $(\mathrm{P}-$ participants/population, C-context, C-concept) was used to formulate the inclusion criteria. ${ }^{61}$

\section{Eligibility criteria}

Participants

This review will consider studies that include persons of any age with a VI with or without additional healthrelated problems. Studies from any geographical location or written in any language will be included.

\section{Concept}

This scoping review aims to identify music interventions used therapeutically in people with a VI to improve wellbeing. It will also analyse the contexts in which the music interventions have been used, and these may include music therapy, music listening and other music-based activities. Therapeutic outcomes and treatment characteristics will be examined. The therapeutic outcome domains will include quality of life (any health-related quality of life measures), physiological outcomes/health related outcomes (eg, blood pressure/ heart rate), mental health (eg, well-being, anxiety, depression) and communication and social outcomes (including social engagement).

\section{Context}

Interventions delivered in all settings will be included, as long as they address the therapeutic outcome domains outlined above.

\section{Types of studies}

The review will include all types of published studies such as, clinical trials, comparative, evaluative and observational. Publication types will include peer-reviewed journal publications, postgraduate theses and conference papers. When the full text of a paper is not available in English language, a certified professional translation service will be used. There will be no publication date restriction. This wide approach to data gathering will provide an extensive and comprehensive selection of sources to address the research question. Studies that have investigated music for non-therapeutic purposes, opinion papers, preconference abstracts, preprints and undergraduate theses will not be included.

\section{Search strategy and information sources}

The databases that will be used are: EMBASE (Ovid interface, 1974 onwards), MEDLINE (Ovid interface, 1948 onwards), CINAHL Plus (EBSCOhost) PsycINFO (EBSCOhost) and Web of Science (Clarivate Analytics). Using a variety of major databases related to: health, life sciences, nursing and psychology will ensure adequate and efficient coverage. ${ }^{62}$ Further search strategies will include free-text hand searches in Google Scholar for grey literature and screening reference lists of all relevant studies. A pilot search was conducted to refine the final search strategy on MEDLINE and CINAHL Plus, using the following selected keywords: 'music' OR 'music therapy' AND 'vision impairment OR 'vision disorder' OR 'eye disease' OR 'blind' to find appropriate search terms as described by Booth. ${ }^{63}$ This process increases the scope of retrieving information relevant to the research questions. ${ }^{63}$ The initial search will be followed by analysis of the text words contained in the titles and abstracts of relevant articles, as well as the index terms used to describe these articles, this will then be adapted for each database search field. The full search strategy for each database will be outlined in the full text of the scoping review.

\section{Selection of sources of evidence}

The identified studies will be exported into the reference manager, Mendeley. Any duplicates will be deleted before exporting to an Excel file. The following information will be recorded in the Excel file: database name, publication title, authors, abstract, export date, search terms used, number of results, grey literature sources searched, other search techniques, limitations and duplicates.

Thereafter, two reviewers (NS and RL) will independently screen all the titles and abstracts for relevance. Each reviewer will evaluate the article against the inclusion criteria. Subsequently, the full version of the relevant articles will be retrieved from Mendeley. Reasons for exclusion of full-text articles will be noted in the Excel file by each author and will be provided as an appendix to the full review. The two reviewers will independently undertake full-text analyses until a final set is agreed on by both. Authors of included papers will be contacted for further information for missing or unclear essential information; specifically, copies of questionnaires or study procedures will be requested if they are not otherwise available. The final set of eligible articles will be held in the Mendeley library. Where there is a disagreement between the two reviewers at any stage of the study selection process, a final agreement will be sought by mutual consensus with input from a third reviewer (PMA). The full review will report the results from the selection of sources of evidence process, which will be summarised using the PRISMA-ScR flow diagram. ${ }^{64}$

\section{Data charting process}

Data will be independently extracted from papers as per the guidelines outlined in the JBI scoping review methodology ${ }^{59}$ A preliminary data extraction table has been devised using the JBI scoping review template ${ }^{57}$ and the Checklist for Reporting Music-based Interventions ${ }^{55}$ (refer to table 2). To minimise reflexivity, that is both researchers involved acknowledge prior experiences, assumptions and beliefs may influence the research process, therefore, the preliminary table will be independently piloted by the two reviewers to check for appropriateness, using a small sample of the included studies. The detailed questions on the data extraction table will be adjusted based on mutual agreement by the two 
Table 2 Preliminary data extraction table

Author/s, year of publication, Journal type (eg: arts, health, neuroscience)

$\begin{array}{ll}\text { Study design } & \text { Study design (eg: experimental design, quasi-experimental design, case study or other)? } \\ \text { Population and Methodology } & \text { How many participants took part in the intervention? } \\ & \text { What were the ocular pathologies and were they congenital or acquired? } \\ & \text { What were the methods of data collection and analysis? }\end{array}$

Therapeutic outcome(s) and corresponding measure(s)
What was the outcome(s) of interest (for example: reduced anxiety, pain and stress or other)?

Did the study meet the desired outcome(s) of interest?

What were the primary and secondary measures: (eg: state trait anxiety inventory, Visual Analogue Scale or other)?

Were other factors reported that contributed to, or influenced, treatment outcomes?

Music-based Intervention Reporting A: Rationale for Music Selection/Intervention Theory

Checklist*

- What was the rationale for the music used and intervention?
B: Intervention Protocols

B.1: Person Selecting the Music

-Was it specified who selected the music (eg: pre-selected by investigator, participant selected)?

B.2: Music

- Was this is an original piece of music or a pre-existing musical composition?

- If a pre-exisiting musical composition was used then was the name of the composer and title of the musical composition stated?

- Was there a description of the music's overall structure (eg: form, elements, instruments or other)?

B.3. Music delivery method

(Live or Recorded)

If the music was played live was it specified who delivered the music and performance?

- Was the size of the performance group specified for the live music (eg: interventionist only, interventionist and participant)?

- If recorded music was used, was placement of playback equipment and/or the use of headphones versus speakers specified?

- If recorded music was used, was the decibel level of music delivered and/or use of volume controls to limit decibels specified?

B.4: Intervention Materials

- Which musical and other materials were specified?

B.5: Intervention Strategies

- What music-based intervention strategies were used (eg: listening, re-creating music by singing/ playing an instrument, instrument/vocal play, improvisation, movement, song writing or other)?

C: Intervention delivery schedule

What was the duration, frequency, and intensity of the treatment?

D: Interventionist

- Were the qualifications and credentials of interventionist(s) reported?

- If more than one interventionist, from which discipline/what qualifications and training details were reported?

E: Treatment fidelity

- Were there any strategies used to ensure that treatment and/or control conditions were delivered as intended (eg: interventionist training, manualised protocols, and intervention monitoring)?

F: Setting

Where was the intervention delivered?

What boundaries were reported (eg, time and location)?

What ambient noise levels were reported in the environment where the intervention took place?

G: Unit of delivery

- Was the intervention delivered to individuals or groups of individuals? reviewers. Any disagreements will be resolved through the involvement of the third reviewer. This procedure will limit subjectivity in the reviewing process. The full review will include the final version of the extracted data table. To ensure quality assurance during the data charting process, both reviewers will read and continue to refer to the JBI review checklist in the reviewer's manual (Methodology for Scoping Reviews, Chapter 11). ${ }^{59}$

\section{Data synthesis and reporting of results}

PRISMA-ScR guidelines will be followed to report the results from the extracted data. ${ }^{60}$ This will allow us to identify the characteristics of sources and map the existing literature. The extracted data will be summarised using percentages where appropriate and will be placed into tables. The categories that will be used for data presentation will be; (1) author and year of publication, (2) study design and country, (3) population and methodology, (4) therapeutic outcomes and corresponding measures and (5) music-based intervention reporting checklist sections (see table 2) A narrative summary will supplement the tabulised results, providing analytical opportunities to identify comparative data related to therapeutic music 
intervention research, undertaken with people with a VI, as per data extraction table 2. The authors anticipate the findings will inform the design of accessible novel music interventions to improve the well-being of people living with a VI.

\section{Patient and public involvement}

This scoping review did not involve patient and public involvement. Therefore consent to participate was not required.

\section{ETHICS AND DISSEMINATION}

This scoping review involves analysis of published scientific literature, therefore research ethics approval was not required. The findings from this scoping review will be submitted to an open access international peer-reviewed journal so that the information can be made freely accessible to researchers, health professionals and the public. Once published, the results will be disseminated at relevant research conferences and communicated via academic social media platforms to expand its reach and utility. Overall, we anticipate that this review will be important for informing future therapeutic music interventions aimed at people with VI, such as the impact of playing music on psychosocial well-being.

Acknowledgements The authors wish to thank the librarians Andrea Packwood from Anglia Ruskin University and Desta Bokre from the Institute of Ophthalmology University College London and for their advice in developing the search strategy.

Contributors NS, PMA, EB and AS conceptualised the study. NS developed the scoping review research questions, methods and drafted the manuscript. NS, PMA, $\mathrm{EB}, \mathrm{RL}$ and $\mathrm{AS}$ critically reviewed and edited the draft and approved the final version of the manuscript.

Funding The authors have not declared a specific grant for this research from any funding agency in the public, commercial or not-for-profit sectors.

Competing interests None declared.

Patient and public involvement Patients and/or the public were not involved in the design, or conduct, or reporting, or dissemination plans of this research.

Patient consent for publication Not applicable.

Provenance and peer review Not commissioned; externally peer reviewed.

Open access This is an open access article distributed in accordance with the Creative Commons Attribution Non Commercial (CC BY-NC 4.0) license, which permits others to distribute, remix, adapt, build upon this work non-commercially, and license their derivative works on different terms, provided the original work is properly cited, appropriate credit is given, any changes made indicated, and the use is non-commercial. See: http://creativecommons.org/licenses/by-nc/4.0/.

ORCID iD

Peter M Allen http://orcid.org/0000-0003-3607-6130

\section{REFERENCES}

1 World Health Organization. Blindness and vision impairment, 2021. Available: https://www.who.int/news-room/fact-sheets/detail/ blindness-and-visual-impairment [Accessed 10 Mar 2021].

2 GBD 2019 Blindness and Vision Impairment Collaborators, Vision Loss Expert Group of the Global Burden of Disease Study. Causes of blindness and vision impairment in 2020 and trends over 30 years, and prevalence of avoidable blindness in relation to vision 2020: the right to sight: an analysis for the global burden of disease study. Lancet Glob Health 2021;9:e144-60.
3 Rafaely L, Carmel S, Bachner YG. Subjective well-being of visually impaired older adults living in the community. Aging Ment Health 2018;22:1229-36.

4 Freeman EE, Muñoz B, West SK, et al. Glaucoma and quality of life: the Salisbury eye evaluation. Ophthalmology 2008;115:233-8.

5 Broman AT, Munoz B, Rodriguez J, et al. The impact of visual impairment and eye disease on vision-related quality of life in a Mexican-American population: proyecto VER. Invest Ophthalmol Vis Sci 2002;43:3393-8.

6 Matthews K, Nazroo J, Whillans J. The consequences of selfreported vision change in later-life: evidence from the English longitudinal study of ageing. Public Health 2017;142:7-14.

7 Brown RL, Barrett AE. Visual impairment and quality of life among older adults: an examination of explanations for the relationship. $J$ Gerontol B Psychol Sci Soc Sci 2011;66:364-73.

8 Rovner BW, Casten RJ. Activity loss and depression in age-related macular degeneration. Am J Geriatr Psychiatry 2002;10:305-10.

9 Pinquart M, Pfeiffer JP. Psychological well-being in visually impaired and unimpaired individuals. Br J Vis Impair 2011;29:27-45.

10 Mojon-Azzi SM, Sousa-Poza A, Mojon DS. Impact of low vision on well-being in 10 European countries. Ophthalmologica 2008;222:205-12.

11 Naylor PD, Labbé EE. Exploring the effects of group therapy for the visually impaired. Br J Vis Impair 2017;35:18-28.

12 Robb SL. Music interventions and group participation skills of preschoolers with visual impairments: raising questions about music, arousal, and attention. J Music Ther 2003;40:266-82.

13 Park HY, Chong HJ, Kim SJ. A comparative study on the attitudes and uses of music by adults with visual impairments and those who are sighted. J Vis Impair Blind 2015;109:303-16.

14 Nyman SR, Dibb B, Victor CR, et al. Emotional well-being and adjustment to vision loss in later life: a meta-synthesis of qualitative studies. Disabil Rehabil 2012;34:971-81.

15 Casten RJ, Rovner BW, Tasman W. Age-Related macular degeneration and depression: a review of recent research. Curr Opin Ophthalmol 2004;15:181-3.

16 Horowitz A, Reinhardt JP, Boerner K. The effect of rehabilitation on depression among visually disabled older adults. Aging Ment Health 2005;9:563-70.

17 Berman K, Brodaty H. Psychosocial effects of age-related macular degeneration. Int Psychogeriatr 2006;18:415-28.

18 Burmedi D, Becker S, Heyl V, et al. Emotional and social consequences of age-related low vision. Vis Impair Res 2002;4:47-71.

19 Stelmack J. Quality of life of low-vision patients and outcomes of low-vision rehabilitation. Optom Vis Sci 2001;78:335-42.

20 Slakter JS, Stur M. Quality of life in patients with age-related macular degeneration: impact of the condition and benefits of treatment. Surv Ophthalmol 2005;50:263-73.

21 Renaud J, Levasseur M, Gresset J, et al. Health-Related and subjective quality of life of older adults with visual impairment. Disabil Rehabil 2010;32:899-907.

22 Mitchell J, Bradley C. Quality of life in age-related macular degeneration: a review of the literature. Health Qual Life Outcomes 2006;4:97.

23 Kempen GIJM, Ballemans J, Ranchor AV, et al. The impact of low vision on activities of daily living, symptoms of depression, feelings of anxiety and social support in community-living older adults seeking vision rehabilitation services. Qual Life Res 2012;21:1405-11.

24 Misajon R, Hawthorne G, Richardson J, et al. Vision and quality of life: the development of a utility measure. Invest Ophthalmol Vis Sci 2005;46:4007-15.

25 Teitelman J, Copolillo A. Psychosocial issues in older adults' adjustment to vision loss: findings from qualitative interviews and focus groups. Am J Occup Ther 2005;59:409-17.

26 Duffy $L$. The experience of patients with age related macular degeneration and the effectiveness of low vision AIDS. Ophthalmic Nursing 1997;1:14-23.

27 Girdler SJ, Boldy DP, Dhaliwal SS, et al. Vision self-management for older adults: a randomised controlled trial. Br J Ophthalmol 2010;94:223-8.

28 Demmin DL, Silverstein SM, Impairment V. Visual impairment and mental health: unmet needs and treatment options. Clin Ophthalmol 2020;14:4229.

29 Dillon L, Tang D, Liew G, et al. Facilitators and barriers to participation in mental well-being programs by older Australians with vision impairment: community and stakeholder perspectives. Eye 2020;34:1287-95.

30 van der Aa HPA, Comijs HC, Penninx BWJH, et al. Major depressive and anxiety disorders in visually impaired older adults. Invest Ophthalmol Vis Sci 2015;56:849-54. 
31 Senra H, Macedo AF, Nunes N, et al. Psychological and psychosocial interventions for depression and anxiety in patients with age-related macular degeneration: a systematic review. Am J Geriatr Psychiatry 2019;27:755-73.

32 Ting DSJ, Krause S, Said DG, et al. Psychosocial impact of COVID-19 pandemic lockdown on people living with eye diseases in the UK. Eye 2021;35:1-3.

33 Bernatzky G, Presch M, Anderson M, et al. Emotional foundations of music as a non-pharmacological pain management tool in modern medicine. Neurosci Biobehav Rev 2011;35:1989-99.

34 Chanda ML, Levitin DJ. The neurochemistry of music. Trends Cogn Sci 2013;17:179-93.

35 Juslin PN, Västfjäll D. Emotional responses to music: the need to consider underlying mechanisms. Behav Brain Sci 2008;31:559-75.

36 Koelsch S, Siebel WA, Fritz T. Handbook of music and emotion: theory, research, applications. Oxford University Press, 2011.

37 Koelsch S. A neuroscientific perspective on music therapy. Ann N Y Acad Sci 2009;1169:374-84.

38 Waldon EG. The effects of group music therapy on mood states and cohesiveness in adult oncology patients. J Music Ther 2001;38:212-38.

39 DeNora T. Music in everyday life. Cambridge: Cambridge University Press, 2000.

40 Snell D, Hodgetts D. Heavy metal, identity and the social negotiation of a community of practice. J Community Appl Soc Psychol 2007;17:430-45.

41 Morgan JP, MacDonald RAR, Pitts SE. "Caught between a scream and a hug": Women's perspectives on music listening and interaction with teenagers in the family unit. Psychol Music 2015;43:611-26.

42 Blood AJ, Zatorre RJ. Intensely pleasurable responses to music correlate with activity in brain regions implicated in reward and emotion. Proc Natl Acad Sci U S A 2001;98:11818-23.

43 de Witte M, Spruit A, van Hooren S, et al. Effects of music interventions on stress-related outcomes: a systematic review and two meta-analyses. Health Psychol Rev 2020;14:294-324.

44 Daly I, Williams D, Malik A, et al. Personalised, multi-modal, affective state detection for hybrid brain-computer music Interfacing. IEEE Trans Affect Comput 2018;11:111-24.

45 Bertelmann T, Strempel I. Short-Term effects of relaxation music on patients suffering from primary open-angle glaucoma. Clin Ophthalmol 2015;9:9.

46 Shue B, Chatterjee A, Fudemberg S, et al. The effects of Mozart's music on the performance of glaucoma patients on automated perimetry. Invest Ophthalmol Vis Sci 2011;52:7347-9.

47 Zhou RX, Li F, Gao K, et al. [Effects of different types of music on intraocular pressure and the underlying mechanism]. Zhonghua Yan Ke Za Zhi 2020;56:25-31.
48 Choi S, Park S-G, Bellan L, et al. Crossover clinical trial of pain relief in cataract surgery. Int Ophthalmol 2018;38:1027-33.

49 Wiwatwongwana D, Vichitvejpaisal P, Thaikruea L, et al. The effect of music with and without binaural beat audio on operative anxiety in patients undergoing cataract surgery: a randomized controlled trial. Eye 2016;30:1407-14.

50 Cruise CJ, Chung F, Yogendran S, et al. Music increases satisfaction in elderly outpatients undergoing cataract surgery. Can $J$ Anaesth 1997;44:43-8.

51 Park HY, Cho MR, Yoon EJ. Research trends of music for people with visual impairments: a review of journals published domestically and internationally from 1998 to 2017. The Journal of the Korea Contents Association 2018;18:243-56.

52 Peters MDJ, Godfrey CM, Khalil H, et al. Guidance for conducting systematic scoping reviews. Int J Evid Based Healthc 2015;13:141-6.

53 Rabiee P, Parker GM, Bernard S. Vision rehabilitation services: what is the evidence? York: University of York, 2015: 1-301.

54 Barrow A, Ting L, Patel V. Creating a holistic support service for people with vision impairment. Br J Gen Pract 2018;68:318.

55 Robb SL, Burns DS, Carpenter JS. Reporting guidelines for musicbased interventions. J Health Psychol 2011;16:342-52.

56 Yinger OS, Gooding LF. A systematic review of music-based interventions for procedural support. J Music Ther 2015;52:1-77.

57 Robb SL, Hanson-Abromeit D, May L, et al. Reporting quality of music intervention research in healthcare: a systematic review. Complement Ther Med 2018;38:24-41.

58 Moore KS, Moore KS. A systematic review on the neural effects of music on emotion regulation: implications for music therapy practice. J Music Ther 2013;50:198-242.

59 Peters M, Godfrey-smith P, Mcinerney P. Guidance for the Conduct of JBI Scoping Reviews. In: Aromataris E, Munn Z, eds. Joanna Briggs institute reviewer's manual. South Australia: Joanna Briggs Institute, 2019. https://reviewersmanual.joannabriggs.org/

60 Page MJ, Moher D, Bossuyt PM, et al. PRISMA 2020 explanation and elaboration: updated guidance and exemplars for reporting systematic reviews. BMJ 2021;372:n160.

61 Khalil H, Peters MD, Tricco AC, et al. Conducting high quality scoping reviews-challenges and solutions. J Clin Epidemiol 2021;130:156-60.

62 Bramer WM, Rethlefsen ML, Kleijnen J. Optimal database combinations for literature searches in systematic reviews: a prospective exploratory study. Syst Rev 2017;6:1-12.

63 Booth A. Unpacking your literature search toolbox: on search styles and tactics. Health Info Libr J 2008;25:313-7.

64 Tricco AC, Lillie E, Zarin W, et al. PRISMA extension for scoping reviews (PRISMA-ScR): checklist and explanation. Ann Intern Med 2018;169:467-73. 